\title{
LOCALLY BOUNDED NONCONTINUOUS LINEAR FORMS ON STRONG DUALS OF NONDISTINGUISHED KÖTHE ECHELON SPACES
}

\author{
FRANCCOISE BASTIN AND JOSÉ BONET
}

(Communicated by Palle E. T. Jorgensen)

\begin{abstract}
In this note it is proved that if $\lambda_{1}(A)$ is any nondistinguished Köthe echelon space of order one and $K_{\infty} \simeq\left(\lambda_{1}(A)\right)_{b}^{\prime}$ is its strong dual, then there is even a linear form : $K_{\infty} \rightarrow \mathrm{C}$ which is locally bounded (i.e. bounded on the bounded sets) but not continuous. It is also shown that every nondistinguished Köthe echelon space contains a sectional subspace with a particular structure.
\end{abstract}

\section{INTRODUCTION}

Following Dieudonné and Schwartz, a locally convex space $E$ is distinguished if its strong dual is barrelled. Grothendieck [5] proved that a metrizable space $E$ is distinguished if and only if $E_{b}^{\prime}$ is bornological. In the theory of Fréchet spaces, the notion of distinguishedness was introduced to avoid some "pathology": the naive idea that the strong dual $E_{b}^{\prime}$ of any Fréchet space $E$, i.e. of a countable projective limit of Banach spaces, must be a countable inductive limit of Banach spaces is false in general. Distinguished Fréchet spaces are those for which the idea really works. In connection with some recent investigations in Fréchet and (DF)-spaces (e.g. applications to weighted inductive limits [2], [3]; consequences of J. Taskinen's negative solution of Grothendieck's "problème des topologies" for $\pi$-tensor products of Fréchet spaces), the class of the distinguished Fréchet spaces and, in particular, the class of the distinguished Köthe echelon spaces, have again become quite important.

The first example of a nondistinguished Köthe echelon space was given by Grothendieck and Köthe [5]. It is a Köthe echelon space of order one $\lambda_{1}(A)$ with the Köthe matrix $A=\left(a_{n}\right)_{n \in \mathbf{N}}$ on the index set $I=\mathbf{N} \times \mathbf{N}$ given by $a_{n}(i, j):=j$ if $i \leq n, a_{n}(i, j):=1$ if $i \geq n+1$. In this case Grothendieck constructed a linear form on $\lambda_{1}(A)_{b}^{\prime}$ which is locally bounded but not continuous (see [5], p. 88). Consequently the topological duals of $\left(\lambda_{1}(A)\right)_{b}^{\prime}$ and of its

Received by the editors May 25, 1989.

1980 Mathematics Subject Classification (1985 Revision). Primary 46A45; Secondary 46A05, 46A07, 46A09.

Key words and phrases. Köthe echelon spaces, Köthe matrices, distinguished spaces, locally bounded linear forms, continuous linear forms. 
associated bornological space do not coincide. An example of Komura (see e.g. [6, p. 292]) shows that there are nondistinguished Fréchet spaces $F$ such that every locally bounded linear form on $F_{b}^{\prime}$ is continuous.

The distinguished Köthe echelon spaces $\lambda_{1}(A)$ have been characterized in terms of the Köthe matrix $A$ in [2] (see also [7] ). We use here this characterization and an adaption of the original argument of Grothendieck based on [3, Appendix p. 194] to show in Theorem 2 that all nondistinguished Köthe echelon spaces $\lambda_{1}(A)$ have the same pathology as the Köthe-Grothendieck example. We also show in Proposition 3 that every nondistinguished Köthe echelon space contains a sectional subspace with a structure similar to the Köthe-Grothendieck example (cf. [7, 4]).

For the notations for Köthe echelon spaces, we refer to [4]. Nevertheless, we recall that we associate with a Köthe matrix $A=\left(a_{n}\right)_{n \in \mathbf{N}}$ on an index (not necessarily countable) set $I$ the decreasing sequence $\mathscr{V}=\left(v_{n}\right)_{n \in \mathbf{N}}$ of strictly positive weights on $I$ defined by $v_{n}(i):=a_{n}^{-1}(i) \quad(n \in \mathbf{N}, i \in I)$. The maximal Nachbin family associated to $\mathscr{V}$ is

$$
\bar{V}:=\left\{\bar{v}: I \rightarrow\left[0,+\infty\left[, \sup _{i \in I} \frac{\bar{v}(i)}{v_{n}(i)}<\infty \text { for all } n \in \mathbf{N}\right\} .\right.\right.
$$

By $[4,2.7]$, the space $\left(\lambda_{1}(A)\right)_{b}^{\prime}$ coincides algebraically and topologically with

$$
K_{\infty}:=\left\{u \in \mathbf{C}^{I}: p_{v}(u):=\sup _{i \in I} \bar{v}(i)|u(i)|<\infty \quad \text { for all } \bar{v} \in \bar{V}\right\}
$$

endowed with the topology defined by the seminorms $\left\{p_{\bar{v}}, \bar{v} \in \bar{V}\right\}$. The bornological space associated to $\left(\lambda_{1}(A)\right)_{b}^{\prime}$ is $k_{\infty}:=\operatorname{ind} l_{\infty}\left(v_{n}\right)$.

We first establish the converse of a result of Bierstedt and Meise [3].

Lemma 1. Let $A$ be a Köthe matrix on an index set I. The following conditions are equivalent:

(a) $\lambda_{1}(A)$ is not distinguished

(b) there are $n \in \mathbf{N}$ and a decreasing sequence $\left(J_{k}\right)_{k \in \mathbf{N}}$ of subsets of I such that for all $k \geq n$

(i) $\inf _{i \in J_{k}} \frac{a_{n}(i)}{a_{k}(i)}>0$ while (ii) there is $l(k)>k$ with $\inf _{i \in J_{k}} \frac{a_{n}(i)}{a_{l(k)}(i)}=0$.

Proof. (b) $\Rightarrow$ (a) is the content of the first appendix in [3, p. 194].

(a) $\Rightarrow$ (b). According to Theorem 2.6. in [2] and to the equivalence of conditions (D) and $(\mathrm{H})$ in [1], there are a sequence $\lambda_{j}>0(j \in \mathbf{N})$ and $n \in \mathbf{N}$ such that for all $\bar{v} \in \bar{V}$ and $m \in \mathbf{N}$ there is $i \in I$ with

$$
\inf \left(\lambda_{1} v_{1}(i), \ldots, \lambda_{m} v_{m}(i)\right) \geq v_{n}(i) \text { and } \bar{v}(i)<v_{n}(i) \text {. }
$$

For every $k \in \mathbf{N}$ we set $J_{k}:=\left\{i \in I: \inf \left(\lambda_{1} v_{1}(i), \ldots, \lambda_{k} v_{k}(i)\right) \geq v_{n}(i)\right\}$. Certainly $\left(J_{k}\right)_{k \in \mathbf{N}}$ is a decreasing sequence of subsets of $I$ and as we have

$$
\inf _{i \in J_{k}} \frac{v_{k}(i)}{v_{n}(i)} \geq \frac{1}{\lambda_{k}} \quad \text { for all } k \in \mathbf{N}
$$

condition (i) is satisfied. 
Suppose there is $k \in \mathbf{N}$ such that (ii) does not hold. Then for all $l>k$ we have

$$
\varepsilon_{l}:=\inf _{i \in J_{k}} \frac{v_{l}(i)}{v_{n}(i)}>0
$$

We define

$$
\alpha_{j}:=\lambda_{j} \quad(j=1, \ldots, k) ; \quad \alpha_{j}:=\frac{1}{\varepsilon_{j}} \quad(j \geq k+1) \quad \text { and } \bar{v}:=\inf _{j \in \mathbf{N}} \alpha_{j} v_{j} .
$$

It is easy to see that $\bar{v} \geq v_{n}$ on $J_{k}$. Now, by $(*)$ there is $i \in I$ such that

$$
\inf \left(\lambda_{1} v_{1}(i), \ldots, \lambda_{k} v_{k}(i)\right) \geq v_{n}(i) \quad \text { and } \quad \bar{v}(i)<v_{n}(i) .
$$

This is a contradiction because the first inequality implies $i \in J_{k}$ and $\bar{v} \geq v_{n}$ on $J_{k}$.

Theorem 2. Let $\lambda_{1}(A)$ be a nondistinguished Köthe echelon space. Then there is a locally bounded noncontinuous linear form on $\left(\lambda_{1}(A)\right)_{b}^{\prime}$.

Proof. Without loss of generality we may assume that condition (b) in Lemma 1 is satisfied with $n=1, l(k)=k+1$ for all $k \in \mathbf{N}$ (this amounts to omit certain $a_{n}^{\prime} s$ if necessary), and we may also suppose that $a_{1}$ is identically 1 (by dividing by $\left.a_{1}\right)$. Then our hypothesis reads as follows: there is a decreasing sequence $\left(J_{k}\right)_{k \in \mathbf{N}}$ of subsets of $I$ such that for all $k \in \mathbf{N}$

$$
\begin{aligned}
& \text { (i) } \varepsilon_{k}:=\inf _{i \in J_{k}} v_{k}(i)>0 \text {; } \\
& \text { (ii) } \inf _{i \in J_{k}} v_{k+1}(i)=0 .
\end{aligned}
$$

For every $l \in \mathbf{N}$ and every sequence $\left(s_{k}\right)_{k \in \mathbf{N}}$ of strictly positive numbers, we set

$$
M\left(l,\left(s_{k}\right)_{k \in \mathbf{N}}\right):=\bigcup_{k \geq l}\left\{i \in J_{k}: v_{k+1}(i)<\frac{1}{s_{k}}\right\} .
$$

We denote by $e_{i}$ the $i$ th unit vector in $\lambda_{1}(A)$. It is easy to see that the family of subsets of $\lambda_{1}(A)$

$$
\left\{e_{i} \in \lambda_{1}(A): i \in M\left(l,\left(s_{k}\right)_{k \in \mathbf{N}}\right)\right\}
$$

is a basis for a filter $\mathscr{M}$ on $\lambda_{1}(A)$. From now on, the space $\lambda_{1}(A)$ will be considered as a subspace of the algebraic dual $K_{\infty}^{*}$ of $K_{\infty}$. Let $\mathscr{U}$ be an ultrafilter in $\lambda_{1}(A)$ containing $\mathscr{M}$.

For every $u \in K_{\infty}$, the family of sets

$$
M\left(l,\left(s_{k}\right)_{k \in \mathbf{N}}, u\right):=\left\{\left\langle u, e_{i}\right\rangle=u(i): i \in M\left(l,\left(s_{k}\right)_{k \in \mathbf{N}}\right)\right\}
$$

is a basis for an ultrafilter $\mathscr{U}(u)$ on $\mathbf{C}$.

Given $u \in K_{\infty}$, there are $M>0$ and $n \in \mathbf{N}$ such that

$$
\sup _{i \in I} v_{n}(i)|u(i)| \leq M \text {. }
$$


If $l>n$ and $s_{k}>0(k \in \mathbf{N})$, we have

$$
\left|\left\langle u, e_{i}\right\rangle\right|=|u(i)| \leq M v_{n}^{-1}(i) \leq M \varepsilon_{n}^{-1}
$$

for all $i \in M\left(l,\left(s_{k}\right)_{k \in \mathbf{N}}\right)$, since $i \in J_{k} \subset J_{n}(k \geq 1)$. Therefore $M\left(l,\left(s_{k}\right)_{k \in \mathbf{N}}, u\right)$ is bounded in $\mathbf{C}$. Then $\mathscr{U}(u)$ converges in $\mathbf{C}$ to an element $f(u)$, from where it follows that the ultrafilter $\mathscr{U}$ converges to $f$ in $\left(K_{\infty}^{*}, \sigma\left(K_{\infty}^{*}, K_{\infty}\right)\right)$.

If $u \in K_{\infty}$ satisfies $\sup _{i \in I} v_{n}(i)|u(i)| \leq \varepsilon_{n}$, then for every $l \geq n$ and every $i \in M\left(l,\left(s_{k}\right)_{k \in \mathrm{N}}\right)$ we have $i \in J_{n}$ thus also $\left|\left\langle u, e_{i}\right\rangle\right|=|u(i)| \leq \varepsilon_{n} v_{n}^{-1}(i) \leq 1$. This implies

$$
|f(u)| \leq 1 \quad \text { for every } u \in \Gamma\left(\bigcup_{n \in \mathbf{N}}\left\{u \in K_{\infty}: \sup _{i \in I} v_{n}(i)|u(i)| \leq \varepsilon_{n}\right\}\right) .
$$

Consequently $f$ is locally bounded and $f$ belongs to $k_{\infty}^{\prime}$.

We prove that $f$ is not continuous on $K_{\infty}$. Indeed: if it were, we could find $\bar{v} \in \bar{V}$ such that

$$
|f(u)| \leq \sup _{i \in I} \bar{v}(i)|u(i)| \quad \text { for every } u \in K_{\infty} .
$$

We may assume that $\bar{v}=\inf _{k \in \mathbf{N}} \alpha_{k} v_{k}$ for certain $\alpha_{k}>0 \quad(k \in \mathbf{N})$. $2^{-1}$.

We first observe that if $i$ belongs to the set $M\left(1,\left(2 \alpha_{k+1}\right)_{k \in \mathbf{N}}\right)$ then $\bar{v}(i) \leq$

Now we define $u(i):=2$ if $i \in M\left(1,\left(2 \alpha_{k+1}\right)_{k \in \mathbf{N}}\right)$ and $u(i):=0$ otherwise. Certainly the sequence $u:=(u(i))_{i \in I}$ belongs to $l_{\infty}\left(v_{1}\right) \subset k_{\infty}=K_{\infty}$. Moreover we have

$$
\sup _{i \in I} \bar{v}(i)|u(i)|=\sup _{i \in M\left(1,\left(2 \alpha_{k+1}\right)_{k \in \mathrm{N}}\right)} \bar{v}(i)|u(i)| \leq 1 .
$$

Therefore $|f(u)| \leq 1$. But $f(u)$ belongs to the closure in $\mathbf{C}$ of the set $\left\{\left\langle u, e_{i}\right\rangle: i \in M\left(1,\left(2 \alpha_{k+1}\right)_{k \in \mathbf{N}}\right)\right\}$ and for every $i \in M\left(1,\left(2 \alpha_{k+1}\right)_{k \in \mathbf{N}}\right)$ we have $\left\langle u, e_{i}\right\rangle=2$. Consequently $f(u)=2$, which contradicts $|f(u)| \leq 1$.

Our next proposition, which is a consequence of Lemma 1, was observed by C. Fernandez.

Proposition 3. Let $A$ be a Köthe matrix on an index set $I$. The following conditions are equivalent:

(a) $\lambda_{1}(A)=\lambda_{1}(I, A)$ is not distinguished.

(b) there is a sectional subspace of $\lambda_{1}(A)$ isomorphic to a Köthe echelon space $\lambda_{1}(\mathbf{N} \times \mathbf{N}, B)=\lambda_{1}(B)$ where the matrix $B=\left(b_{n}\right)_{n \in \mathbf{N}}$ satisfies

(1) $b_{n}(k, j)=b_{1}(k, j) \quad$ for $n \leq k$ and

(2) $\lim _{j \rightarrow \infty} \frac{b_{n}(n, j)}{b_{n+1}(n, j)}=0 \quad$ (cf. $\left.[7,4]\right)$. 
Proof. (b) implies (a) as a consequence of [7, 4] where it is proved that these two conditions on $B$ imply that $\lambda_{1}(B)$ is not distinguished.

For the converse we apply Lemma 1 and we proceed as in the proof of Theorem 2 to find a decreasing sequence $\left(J_{k}\right)_{k \in \mathbf{N}}$ of subsets of $I$ such that

$$
\begin{aligned}
& \text { (1) } a_{1}(i)=1 \text { for all } i \\
& \text { (2) } \varepsilon_{k}:=\inf \left\{a_{k}^{-1}(i): i \in J_{k}\right\}>0, \\
& \text { (3) } \inf \left\{a_{k+1}^{-1}(i): i \in J_{k}\right\}=0 \quad \text { for all } k \in \mathbf{N} \text {. }
\end{aligned}
$$

By (2) and (3), given $k \in \mathbf{N}$ we can find a sequence $(i(k, j))_{j \in \mathbf{N}}$ of different elements of $J_{k} \backslash J_{k+1}$ such that $\lim _{j \rightarrow \infty} a_{k+1}^{-1}(i(k, j))=0$. Now we define $J:=\{i(k, j): k, j \in \mathbf{N}\}$ and the Köthe matrix $B=\left(b_{n}\right)_{n \in N}$ by $b_{n}(k, j):=$ $a_{n}(i(k, j))$ if $k<n$ and by $b_{n}(k, j):=1$ if $k \geq n$. By construction, $\lambda_{1}\left(J, A_{\mid J}\right)$ is a sectional subspace of $\lambda_{1}(I, A)$ and it is easy to see that $B$ satisfies conditions (1) and (2). Moreover, the canonical map

$$
\Psi: \lambda_{1}\left(J, A_{\mid J}\right) \rightarrow \lambda_{1}(\mathbf{N} \times \mathbf{N}, B) \quad y \mapsto(y(i(k, j)))_{j, k \in \mathbf{N}}
$$

is a topological isomorphism. Indeed, as we have $b_{n}(k, j) \leq a_{n}(i(k, j))$ for every $k, j \in \mathbf{N}$, this map is well defined and continuous. Using condition (2) above and the definition of $B$, it is also direct to prove that $\Psi^{-1}$ is well defined and continuous. Thus the proof is complete.

Remark 4. Our theorem provides a class of examples which are relevant from another point of view. In fact, if $\lambda_{1}(A)$ is any nondistinguished Köthe echelon space, then $\left(\lambda_{1}(A)^{\prime \prime}, \sigma\left(\lambda_{1}(A)^{\prime \prime}, \lambda_{1}(A)^{\prime}\right)\right)$ is quasi-Suslin but not $K$-Suslin.

Indeed, this space is quasi-Suslin by [6, Chap. 1, 4.3.(23)] and it is not $K$ Suslin by $[6$, Chap. 1, 4.3.(24)] if we observe that according to Theorem 2 , the space $\left(\lambda_{1}(A)^{\prime}, \mu\left(\lambda_{1}(A)^{\prime}, \lambda_{1}(A)^{\prime \prime}\right)\right)$ is not bornological, hence not barrelled.

\section{REFERENCES}

1. F. Bastin, On bornological $C \bar{V}(X)$ spaces, Arch. Math. 53 (1989).

2. K.-D. Bierstedt and J. Bonet, Stefan Heinrich's density condition for Fréchet spaces and the characterization of the distinguished Köthe echelon spaces, Math. Nachr. 135 (1988), 149-180.

3. K.-D. Bierstedt and R. Meise, Distinguished echelon spaces and the projective description of weighted inductive limits of type $\mathscr{V}_{d} C(X)$. Aspects of mathematics and its applications, North-Holland Math. Library (1986), 169-226.

4. K.-D. Bierstedt, R. Meise and W. H. Summers, Köthe sets and Köthe sequence spaces, Functional analysis, holomorphy and approximation theory, North-Holland Math. Stud. 71 (1982), 27-91.

5. A. Grothendieck, Sur les espaces $(F)$ et $(D F)$, Summa Brasil Math. 3 (1954), 57-122. 
6. M. Valdivia, Topics in locally convex spaces, North-Holland Math. Stud. 67 (1982).

7. D. Vogt, Distinguished Köthe spaces, Math. Z. 202 (1989), 143-146.

Aspirant FNRS, Université de Liège, Institut de Mathématique, 15 avenue des Tilleuls, B-4000 Liège, Belgium

Universidad Politécnica de Valencia, Departamento de Matemática Aplicada, E-46071 Valencia, Spain 